\author{
Breezy Vizeu \\ FAPAR - Faculdade Paranaense, Brasil \\ breezyvizeu@yahoo.com.br
}

\title{
La formación de la cultura jurídica brasilera en la segunda mitad del siglo XIX ${ }^{1}$
}

\begin{abstract}
The purpose of this study is to document the existence of an effective Brazilian juridical culture which arose during the second half of the nineteenth century. In order to attain our objective, it was important to search for the distinguished jurists of this period, to examine their academic training and their writing, as well as to understand how they were influenced by the scientific production and training of the time. In addition, we investigated the existence of the university faculties in this period and the training of lawyers in this country. In this context, an analysis of this period revealed that, the fact that the law was not codified in the nineteenth century, did not mean that the old Portuguese legal system was maintained unchanged in Brazil. The profusion of laws in the private sector contributed as much to regulating the specific problems of our society as to defining the boundaries of our legal culture in the second half of the nineteenth century.
\end{abstract}

Key words: Nineteenth century, right, history, juridical culture, juridical teaching.

\section{Introducción}

La presente investigación tuvo por objetivo reflexionar acerca de la existencia de una efectiva cultura jurídica brasilera en la segunda mitad del siglo XIX; para lograr dicho objetivo, indagamos cuáles fueron las efectivas contribuciones para la formación de esta cultura jurídica en nuestro país. Por lo tanto, partimos del presupuesto de que el derecho constituye un fenómeno social, es decir, es fruto de

1 Este artículo fue traducido del portugués al español por Héctor Segura Ramírez. 
una construcción a partir de relaciones sociales entre personajes inmersos en una determinada sociedad y en un tiempo específico. Así, al proponerse el estudio del derecho, este debe ser hecho a partir de dos presupuestos condicionantes en los cuales está inmerso. Sobre este asunto, considera Ricardo Marcelo Fonseca que:

El análisis de los institutos, conceptos o teorías solamente puede ser efectuado a partir de sus inscripción en un determinado tiempo, considerando todos los condicionamientos sociales, económicos, políticos, mentales, etc., que los circundan, delimitan y condicionan (Fonseca, $2000: 574){ }^{2}$

Tales condicionantes, de hecho, son importantes para el estudio del período histórico escogido, en nuestro caso la segunda mitad del siglo XIX, en el contexto de la realidad brasilera, por los importantes y significativos acontecimientos. De hecho, en breves líneas, apenas para citar aquellos acontecimientos que tuvieron mayor impacto en la vida de las personas de este período, tenemos: en el ámbito político, la Independencia, en el año de 1822, y la proclamación de la república, en el año de 1889; ya en el ámbito jurídico, tenemos la promulgación de dos Constituciones: la Constitución Imperial, en 1824, y la Constitución de la República, en 1891, así como la promulgación del Código Criminal, en el año de 1830, y del Código Comercial, en el año de 1850.

Sin embargo, a pesar de la importancia de tales acontecimientos para el Estado brasilero, el siglo XIX se muestra muy peculiar cuando intentamos comprender el funcionamiento de su estructura jurídica, así como el funcionamiento de las fuentes disponibles. Así, todo indica que el período relativo a este siglo se muestra como un período de transición entre lo pre-moderno y el absolutismo jurídico; en otros términos, lo pre-moderno en el esfuerzo de modernización jurídica

Hechas estas consideraciones iníciales, la propuesta de la reflexión acerca de la existencia o no de una cultura jurídica brasilera ${ }^{3}$ en la segunda mitad del siglo XIX tuvo como parámetros el análisis de cuáles fueron las efectivas contribuciones para la formación de esta cultura jurídica en nuestro país. Teniendo en cuenta este objetivo, fue realizada una búsqueda de los juristas destacados en este periodo, de su formación académica, de sus obras, así como

2 Sobre la importancia de la inserción del derecho en un determinado tiempo y espacio, António Manuel Hespanha afirma que "el derecho existe siempre 'en sociedad' (situado, localizado) y que, cualquiera que sea el modelo usado para describir sus relaciones con los contextos sociales (simbólicos, políticos, económicos, etc.), las soluciones jurídicas son siempre contingentes en relación a un dado entorno (o ambiente). Son en este sentido, siempre locales" (Hespanha, 2005 : 21).

3 Es importante resaltar que la cultura jurídica que en este tópico nos proponemos buscar consiste en una cultura jurídica genuinamente brasilera, disociada de la influencia extranjera. Para este estudio, tomamos como parámetro, especialmente, el estudio de Fonseca (2006a; 2006b). 
de la influencia recibida para su formación por la producción científica de la época. En este estudio será importante, también, considerar la existencia de las facultades de derecho en este periodo y la formación de los abogados en nuestro país. En síntesis, para el objetivo de este estudio fueron realizadas dos modalidades de análisis: los cursos jurídicos y los profesionales del ámbito jurídico, así como la producción científica y legislativa en la segunda mitad del siglo XIX en este país.

\section{Formación de la cultura jurídica en la segunda mitad del siglo XIX y la educación jurídica en el Brasil}

En este sentido, para dar inicio a tal empresa es importante recordar que, hasta la Independencia, en el año de 1822, el aparato jurídico fue impuesto por la metrópoli portuguesa; de esta manera, toda la legislación existente hasta aquel momento era oriunda de Portugal. Basta recordar que las Ordenaciones Filipinas, del año 1603, todavía continuaban en vigor ${ }^{4}$ en el Brasil (y continuaría por más de tres siglos, inclusive resistiendo después de la promulgación de la República, en el 1889, con presencia en la Constitución brasilera de la República). ${ }^{5}$ Así, a partir de la Independencia, el Brasil se vio obligado a organizarse a partir de la legislación portuguesa trasplantada aquí por imposición de la Corona. Es en este escenario, que el Brasil independiente comienza a dar sus primeros pasos, a construir su propia historia jurídica independiente de Portugal.

Sin embargo, a pesar de haber alcanzado la Independencia, el Brasil necesitaba de más tiempo para consolidar y construir su propio aparato jurídico. Así, el 20 de octubre de 1823 es promulgada una ley determinando la observancia de las ordenaciones, leyes, regimientos, licencias, decretos y resoluciones hasta

4 Tal hecho realmente merece destacarse, por su peculiaridad: ¿cómo una legislación ultrapasada, que ni siquiera en su país de origen permaneció tanto tiempo en vigor, duró tanto tiempo en nuestro país? Sobre tal peculiaridad, argumenta Pontes de Miranda: "Destino singular el de las Ordenaciones Filipinas: hechas y promulgadas bajo reyes intrusos, que no figuran en las galerías, como si la historia todavía insistiese en no reconocer la usurpación española, fueran revalidadas y confirmadas por el nuevo rey nacionalista (1643) y victorioso; y - en el suelo americano - con la Independencia del Brasil y, después, con la República, dos veces se confirmaron y persistieron. Como en 1640, el Código de 1603 resistió al rompimiento de los lazos políticos entre Portugal y el Brasil, que se hizo independiente, y todavía después de revocado en Portugal, al desmoronamiento del trono imperial brasilero. Es de verdad admirable que la codificación considerada de 'innecesaria, intempestiva y publicada con dolo' (Lei de 25 de mayo de 1773), 'superflua y maquinada por astutos e infieles compiladores movidos por mutuos y particulares intereses', según decía otra Ley, la de 25 de enero de 1775, permaneciese en vigor, fuera de Europa, de 1603 hasta el 31 de diciembre de 1916. Resistió a tres mudanzas políticas radicales - la de 1640, la de 1822 y la de 1889" (Miranda, 1981 : 42).

5 El artículo 83 de la Constitución de la República así lo disponía: "Continúan en vigor hasta que no sean revocadas las leyes del antiguo régimen, en lo que explícita o implícitamente no sea contrario al sistema de gobierno afirmado por la Constitución y por los principios en ella consagrados". 
el 25 de abril de 1821, en cuanto no fuese confeccionado un Código, o hasta cuando tales diplomas normativos no fueran alterados. Además, la Constitución Imperial brasilera del 25 de marzo de 1824 determinó, en su artículo 179, ${ }^{6}$ XVIII, la organización de un Código Civil, fundado en las bases sólidas de la justicia y de la equidad, reconociendo las Ordenaciones Filipinas ${ }^{7}$ como un ordenamiento jurídico brasilero hasta la promulgación de un Código Civil. Así, este hecho comprobaba que el inicio del proceso de construcción jurídica del Brasil independiente tuvo como punto de partida la cultura jurídica portuguesa existente hasta entonces, y el esfuerzo de superación de esta independencia, así como el esfuerzo para el nuevo Estado independiente de construir su propio aparato jurídico, y, a partir de allí, su propia cultura jurídica.

Así, la tarea de construcción de una cultura nacional parece no haber sido de las más fáciles. Basta recordar dos factores importantes que dificultaron mucho más esta consolidación cultural en nuestro país: en primer lugar, vale la pena recordar, no existía ninguna Facultad de derecho hasta la Independencia; en segundo lugar, y como consecuencia del primer factor, es decir, de la ausencia de cursos superiores, nuestros abogados eran todos formados por la Facultad de Coímbra. Tales factores, como lo veremos con mayor profundidad en este tópico, tornaron más difícil la delimitación de los contornos de una cultura brasilera, hasta en la primera mitad del siglo XIX. En este sentido, se trata de un momento de transición, en el cual las circunstancias no se muestran tan favorables ${ }^{8}$, lo que sin embargo no constituyó un obstáculo insuperable para la búsqueda de esta conquista, como veremos más adelante.

6 La constitución del Imperio del Brasil así prescribió, en su artículo 179, № XVIII: "Se organizará, cuanto antes, un Código Civil y uno Criminal, fundado en las sólidas bases da justicia y la equidad." 7 Es importante señalar el hecho de que las Ordenaciones Filipinas tuvieron vigencia por más tiempo en nuestro país que en Portugal. De hecho, a pesar de colonia y metrópoli haber caminado juntas durante un buen tiempo, después de la Independencia del Brasil, ambas comienzan a caminar caminos diferentes. En este sentido, Portugal, influenciado por las ideas iluministas de la codificación napoleónica, promulga su Código Civil en 1867. El Brasil, a pesar de necesidad de la reformulación legislativa, solo consiguió su Código Civil medio siglo después, con poca influencia de la codificación francesa.

8 De hecho, Ricardo Marcelo Fonseca refleja que, especialmente en la primera mitad del siglo XIX, se torna difícil definir los contornos de una "cultura jurídica brasilera"; al mismo tiempo, pondera que "progresivamente es perceptible el hecho de que la tradición europea recibida en el Brasil va siendo progresivamente trabajada, burilada y adaptada a las inúmeras particularidades y contradicciones vividas por el joven Estado, de modo que dio contornos no arbitrarios y bastante típicos al derecho. Y no podía ser diferente: el Brasil entra en la época liberal como un país que busca estructurase jurídica y políticamente en un contexto de tensión. Por un lado, están presentes las sombras del viejo mundo del antiguo régimen que, en el caso brasilero, implican una estructura social colonial profundamente centralizada, oligárquica y montada en la explotación a partir del uso masivo del trabajo esclavo. En el ámbito jurídico, esta estructura social particular es aprobada 
De hecho, cuando el Brasil alcanzó su Independencia, hasta entonces no había ninguna Facultad de derecho9 instalada en nuestro territorio, al contrario de la América española que ya contaba con 23 universidades.10 Este hecho se debe a intereses de la propia Metrópoli, que tenía como política una formación centralizada con el objetivo de mantener una masa de personas alienadas y sin ninguna formación, de modo que el establecimiento de escuelas superiores perturbaría su interés de una formación centralizada, es decir, "el establecimiento de universidades en el Brasil (y como se ve, de modo particular, la creación de facultades de derecho) era considerado una amenaza al dominio colonial" Fonseca, (2006b : 339-369).

Luego, después de la independencia, comenzó un largo debate legislativo para la instalación de Facultades en nuestro país, para formar una élite independiente, desvinculada de los moldes del modelo colonial. De esta manera, la idea era

sustituir la hegemonía extranjera -fuera ella francesa o portuguesapor la creación de establecimientos de enseñanza de calidad, como las escuelas de derecho, que se responsabilizarían por el desarrollo de un pensamiento propio y darían a la nación una nueva Constitución (Schwarcz, 93: 141-188).

Así, en este contexto social, de una voluntad de consolidación de ideas propias, en 1828 se inician los primeros cursos de derecho; con el proyecto de ley del 31 de agosto de 1826, convertido en ley el 11 de agosto 1827, se dispuso sobre la formación de dos Facultades ${ }^{11}$ para atender dos conjuntos de población: una para atender la región Norte, representada por la Facultad de

con la vigencia de las viejas Ordenaciones Filipinas y de la legislación portuguesa colonial (sobre todo en el derecho privado) (Fonseca, 2006b).

9 En realidad, es solo en el año de 1808, en virtud de las invasiones napoleónicas, cuando la familia real portuguesa llega y establece la corte en el Brasil, que fue permitida la instalación de escuelas superiores en nuestro territorio; sin embargo, como será visto a continuación, la primera Facultad de derecho solo será instalada en 1827, después de un largo debate legislativo, luego de la Independencia del Brasil.

10 Tal afirmativa puede ser encontrada en las palabras de José Murilo de Carvalho: "En contraste con España, Portugal nunca permitió la creación de universidades en su colonia. Al final del período colonial habían por lo menos 23 universidades en la parte española de América, tres de ellas en México. Unas 150 mil personas habían sido formadas en esas universidades. Solo la Universidad de México formó 39.367 estudiantes. En la parte portuguesa, escuelas superiores solo fueron admitidas después de la llegada de la corte en 1808. Los brasileros que quisieran y pudiesen seguir un curso superior tenían que viajar a Portugal, sobre todo a Coímbra. Entre 1772 y 1872, pasaron por la Universidad de Coímbra 1242 estudiantes brasileros. Comparado con los 150 mil de la colonia española, el número es ridículo" (Carvalho, 02: 23). Sobre este asunto ver: Cunha (1980). 11 Según Lilia Moritz Schwarcz, de las dos Facultades existentes, San Pablo tuvo una mayor influencia en el modelo político liberal, ya que la facultad de Recife recibió influencia de las escuelas darwinista social y evolucionista, teniendo como objetivo su preocupación con el análisis social así como el carácter doctrinador de sus intelectuales (Schwarcz, 1993). 
Olinda, después transferida en el año de 1854 para Recife; y otra facultad para atender la región Sur, localizada en la ciudad de San Pablo.

Es a partir de este escenario, con la instalación de dos facultades de derecho, ${ }^{12}$ que comienzan a ser delineados los primeros contornos de nuestra cultura jurídica, en un proceso de lenta e insistente transformación. Efectivamente, a pesar de haber dado el primer paso de emancipación, en la primera mitad del siglo XIX, de acuerdo con Ricardo Marcelo Fonseca, la cultura jurídica brasilera era formada "por un puñado de hijos de las élites con formación en la Universidad de Coímbra y otro contingente (ciertamente no significativo) de estudiantes formados a partir de la década de los 30 en los jóvenes y pragmáticos cursos de derecho de Recife y San Pablo" (Fonseca, 2006: 71). Así, la primera mitad del siglo XIX aparece como un periodo de transición, donde las cosas ya comenzaron a suceder; hay una voluntad en este sentido, pero el proceso es lento. Es el caso de esos primeros cursos jurídicos que, en el inicio, estaban más preocupados por la formación de una élite apta substituir a la antigua burocracia oriunda de Portugal que con la formación de juristas preocupados con lo social, con los problemas de su tiempo. En este sentido, acerca de la formación del abogado en el Brasil, Sergio Adorno concluye que:

Desde muy temprano, los cursos jurídicos nacieron orientados mucho más por la preocupación de constituir una elite política unida, disciplinada, devota a las razones del Estado, que se pusiera al frente de los negocios públicos y pudiese, poco a poco, substituir la tradicional burocracia heredada de la administración joanina, de que por la preocupación en formar juristas que produjesen la ideología jurídico-política del Estado Nacional emergente (Adorno, 88: 236). ${ }^{13}$

34 Así, todo parece indicar que en la primera mitad del siglo XIX los primeros cursos jurídicos instituidos en nuestro país estaban mucho más preocupados por las necesidades políticas del Estado que por los problemas sociales o por la

12 Según Antonio Carlos Wolkmer, la formación de los cursos jurídicos en el Brasil tuvo como elementos norteadores el individualismo y el liberalismo económico: "la implementación de los dos primeros cursos de derecho en el Brasil, en 1827, uno en San Pablo y otro en Recife, reflejó la exigencia de una sucesora de la dominación colonizadora, que buscaba concretizar la Independencia político-cultural, recomponiendo ideológicamente la estructura de poder y preparando una nueva camada burocrático-administrativa, sector este que asumiría la responsabilidad de gerenciar el país (Wokmer, 2003: 80).

13 Tratando acerca de la profesionalización de la política, el autor afirma: "la profesionalización de la política, iniciada en el interior de las academias de derecho, otorgó un papel determinante al abogado. Operando en el contexto de una monarquía patrimonial, los abogados se apropiaron de las oportunidades de acceso y promoción en las carreras directivas de los órganos centrales y regionales del gobierno" (Adorno, $1988: 78$ ). 
formación de juristas que contribuyesen a la formación de una cultura jurídica genuinamente brasilera, es decir, sin que la misma estuviera impregnada de las ideas de la cultura jurídica europea, especialmente portuguesa. En este sentido, Ricardo Marcelo Fonseca concluye que en este período: "no hubo un ambiente intelectual adecuado para la formación de una cultura jurídica nacional sólida y que se puede afirmar con vigor la existencia de una tipicidad que la distinguiese de la herencia portuguesa" (Fonseca, $2006: 339) .{ }^{14}$

Es importante señalar que, en esta primera mitad del siglo XIX, el inicio de las actividades de las dos facultades de derecho no fue nada fácil; en realidad, este inicio fue marcado por varios problemas con las instalaciones, toda vez que la estructura física se constituía de casonas muy viejas; además, este inicio estuvo marcado, por un lado, por la falta de respeto de los alumnos; por el otro, por la falta de autoridad de los profesores, en un ambiente "marcado sobre todo por la poca importancia de su contribución y por la pronunciada influencia de la Iglesia" (Schwarcz, 1993: 144). A pesar de los problemas comunes entre las dos Facultades, la de Recife ${ }^{15}$ quedó marcada especialmente por preparar y conducir la formación de doctrinadores en el ámbito del derecho nacional: la de São Paulo, ${ }^{16}$ por su parte, fue responsable por la formación de grandes políticos y burócratas del Estado.

14 En efecto, varios autores han relatado la forma precaria de la educación en la primera mitad del siglo XIX. En este sentido, Sérgio Adorno afirma que: "Las permanentes críticas dirigidas contra la mala calidad de la educación y contra la propia habilitación del cuerpo docente, formuladas hasta por académicos que vivieron ese proceso educativo en aquella época, sugieren que la profesionalización del abogado se operó fuera del contexto de las relaciones didácticas establecidas entre el cuerpo docente y el cuerpo discente, a pesar de las doctrinas jurídicas difundidas en el salón de clase" (Adorno, 1988 : 93). Todavía sobre la crítica de la educación en este período, Américo Jacobina Lacombe, citado por Venâncio Filho, así concluyó: "Que las facultades fueron focos de cultura es verdad, como se ve en la 'Escuela de Recife', que es un hecho. Pero nunca enseñaron, lo que es otra cosa" (Venâncio, 1982: 164).

15 De hecho, según Lilia Moritz Schwarcz, la facultad de Recife, inaugurada en 1828, representó la penetración directa de las viejas ideas portuguesas, las costumbres, en la mayoría de los profesores, como en buena parte de los alumnos. Así, todo recordaba la escuela de Coímbra, inclusive los trajes. La facultad, con sede inicial en Olinda, en el inicio de su existencia quedó marcada por su poca contribución y por la influencia de la Iglesia. Además, los profesores, debido a la baja remuneración que recibían, acababan relajándose, y se ausentaban con frecuencia. Las ausencias de los alumnos también eran frecuentes (Schwarcz, 1993).

16 En relación con las características de la Academias de San Pablo, afirma Sérgio Adorno: "Si la história de la Academia de San Pablo hace sobresalir la ausencia de una efectiva educación jurídica en el Imperio, que apenas produjo juristas de notoriedad nacional y doctrinadores del derecho, hace también destacar su lado reverso: fue cantera de un verdadero "mandarinato imperial" de abogados. Bajo esta perspectiva, el estudio de las condiciones sociales, culturales e intelectuales que inician la formación del abogado en San Pablo se impone como requisito para desenredar los nexos sociológicos entre los intelectuales brasileros en el Siglo XIX, la organización de la cultura jurídico-política, la profesionalización de la actividad política en el horizonte del liberalismo y, 
En este sentido, de acuerdo con las contingencias vividas en el inicio de sus instalaciones por las dos Facultades de derecho, y por las inúmeras dificultades por las que pasaron, no es posible visualizar una cultura jurídica en esta primera mitad del siglo XIX, que se dijera divorciada de las ideas e influencias de la antigua Metrópoli. Además, tal cuadro comienza a dar señales de mudanza a partir de la segundad mitad del siglo XIX, con la edición de la reforma de la educación, en 1854, en un periodo marcado también por la transferencia de la Facultad de Olinda para Recife, ${ }^{17}$ cuando comienzan a surgir nombres importantes para la construcción de la doctrina jurídica nacional; ${ }^{18}$ siendo así, "no será por mera coincidencia que el inicio de la segundad mitad del siglo XIX pase a ser señalado como el de la consolidación y apogeo del Imperio, correspondiendo también a una fase de grandes transformaciones jurídicas" (Venâncio, 1982 : 64).

En efecto, la Reforma de la educación instituida en 1854 (Decreto 1386) objetivaba poner orden en los cursos con la intención de contener la desobediencia que se había instalado en el ambiente académico, instituyó exámenes preparatorios, impuso calendarios más rígidos, limitó el número de reprobaciones, y teniendo como objetivo poner fin a los casos de violencia registrados en el interior de las facultades, "fue instaurado un riguroso sistema de castigos, pudiendo la punición ir de una simple reprimenda a la expulsión de clase por el profesor, o a la prisión correccional por el director" (Schwarcz, 1993 : 147). Otro punto significativo fue el registro de varias propuestas de reforma curricular. ${ }^{19}$ Todos estos acontecimientos contribuyeron para la formación de la cultura jurídica nacional.

finalmente, la génesis del modelo de ciudadanía en esa sociedad, durante el surgimiento del orden social competitivo (Adorno, $1988: 79$ ).

17 De hecho, la transferencia de la Facultad para Recife en 1854 representará "un cambio tanto geográfico como intelectual. Es solo a partir de entonces que se puede pensar en una producción original y en la existencia de un verdadero centro creador de ideas y aglutinador de intelectuales comprometidos con los problemas de su tiempo y de su país" (Schwarcz, 1993 : 146-147).

18 Sobre el surgimiento de estos intelectuales, Lilia Moritz Schwarcz concluye: "Pero, si la transferencia no resultó en un mejoramiento de la instalaciones, lo mismo no se puede decir de la producción intelectual. Es a partir de ese momento que se percibe el surgimiento de un nuevo grupo de intelectuales, cuya producción traspasará los estrechos límites regionales (Schwarcz, 1993). 19 Como un ejemplo de tales propuestas, tenemos la reforma de reforma de 1879 "que establece la 'educación libre', abole la obligatoriedad de la frecuencia y divide el curso en dos secciones distintas: ciencias jurídicas y ciencias sociales'. A partir de esta fecha, al programa de 'ciencias jurídicas' corresponderían los cursos de derecho natural, romano, constitucional, civil, criminal, comercial, legal, teoría y práctica del proceso. El curso de ‘ciencias sociales' sería compuesto por las cátedras de derecho natural, público, universal, constitucional, eclesiástico, de las gentes, administrativos, y diplomacia, historia de los tratados, ciencia de la administración, higiene pública, economía, política" (Schwarcz, 1993 : 147). 
Al lado de los acontecimientos narrados anteriormente, surgía una nueva figura de intelectuales, más preocupados con el estudio científico del derecho, alejándose de las convicciones de los primeros juristas, influenciados por la orientación y religiosa metafísica. De estos últimos, merecen destaque dos grandes profesores de la escuela de São Paulo: José María de Avelar Brotero (1829), y José María Correia de Sá e Benevides (1887). En la facultad de Recife, José Soriano de Souza (1871) a pesar de haber ingresado en la facultad apenas en 1891, sus teorías se asemejan a las de los profesores arriba mencionados, con una fuerte carga de pensamiento religioso. En este sentido, este primer período de la cultura jurídica brasilera acaba siendo "embutido en una concepción escolástica de derecho natural. Se nota, así, que la 'modernidad' brasilera, cuya Constitución es tan deseada desde la Independencia, acaba siendo introducida "por la mitad" (Fonseca, 2006b : 339-369)

Por otro lado, en la segunda mitad del siglo XIX, según Lilia Moritz Schwarcz, especialmente en la década de 70 de este siglo, comenzaran a surgir nuevas ideas en el ambiente académico, que vienen a cuestionar la teoría del derecho natural hasta entonces difundido; se trata, de una propuesta, de una visión laica del mundo, en oposición al pensamiento religioso, ${ }^{20}$ representando, en este sentido, un marco para la historia cultural brasileira, en la medida en que "representa el momento de la entrada de todo un nuevo ideario positivista evolucionista de base racional" (Fonseca, 2006b : 339-369). Varios juristas fueron representativos para esta nueva generación de ideas como Silvio Romero, Tobías Barreto, Francisco de Paula Batista (1872), Teixeira de Freitas, Lafayette Rodrigues Pereira. Para afirmar esta premisa, será tratada a seguir, en breves líneas, la contribución en la construcción de la cultura jurídica nacional, así como el pensamiento de cada uno de los autores mencionados.

De esta forma, damos inicio a este asunto comenzando con Silvio Romero. De acuerdo con Lilia Moritz Schwarcz (1993), Silvio Romero fue ejemplo de "homem de sciencia"; su producción se destacó por el radicalismo de las posiciones, el apego al positivismo francés, viendo en el mestizaje la solución para la posible homogeneidad nacional, basando su solución en una postura teórica que encontraba en el "criterio etnográfico" el modo de desvelar los problemas nacionales, a pesar de no defender la igualdad de las razas. De cualquier forma, es a partir de Silvio Romero que el derecho pasa a combinarse con la antropología, gana status de "sciencia" y tiene el derecho de hablar y determinar los destinos y los problemas de la nación. Silvio Romero tiene el derecho de hablar y determinar los destinos y los problemas de la nación. Es importante destacar que Silvio Romero fue uno de los representantes de esa

20 De hecho, según esta autora: "[...] a partir de ese momento toma fuerza un movimiento de contestación a la teoría del derecho natural en el cual el orden social era comprendida como absolutamente rígida e inmutable" (Schwarcz, 1993 : 150). 
generación responsable de esa mudanza teórica, y que también contribuyó para la formación de nuestra cultura jurídica. Citación interesante para demostrar tal afirmativa es el prefacio que el autor escribe al libro de Tobías Barreto:

El decenio que va de 1868 a 78 es el más notable de cuantos en el siglo XIX constituyeron nuestra vida espiritual... De repente la inmutabilidad de las cosas mostró [...] Un conjunto de ideas nuevas se agitó sobre nosotros de todos los puntos del horizonte [...] Positivismo, evolucionismo, darwinismo, crítica religiosa, naturalismo, cientificismo en la poesía y en la novela, nuevos procesos de crítica e historia literaria, transformación de la instrucción del derecho y de la política, entonces todo se agitó y el grito de alarma emergió de la Escuela de Recife. (Schwarcz, 1993 : 148).

En efecto, en este prefacio, Silvio Romero representa muy bien las nuevas ideas de esa nueva generación que viene, por lo tanto, a romper con los antiguos padrones de los primeros tiempos de instalación de las facultades de derecho en nuestro país, representando, por lo tanto, una mudanza teórica y cultural para el Brasil en el siglo XIX.

Otro autor destacado en el escenario jurídico nacional en la segunda mitad del siglo XIX fue Tobías Barreto, autor de varias obras de carácter científico y otras de literatura y filosofía; fue combatiente declarado de la metafísica, conforme se desprende de sus propias palabras:

Con excepción de media docena de ignorantes o de perezosos que una vez escondidos y resguardados dentro de la cueva de una vieja teoría, hecha y acabada, nunca más salen de la choza y con la cabeza canosa, hoy nadie está dispuesto a perder inútilmente su tiempo con las infelices investigaciones de la metafísica jurídica (Bareto, 1962 : 77).

En seguida critica a los autores del derecho natural:

Es necesario de una vez por todas acabar con semejantes antigüedades. El derecho es una obra del hombre, al mismo tiempo una causa y un efecto del desarrollo humano. La historia del derecho es una de las formas de historia de la civilización. Los tercos teoristas de un derecho natural son figuras anacrónicas, están fuera de su tiempo. Si ellos poseyesen ideas más claras sobre la historia de tal derecho, no se atreverían, todavía hoy, a tener en cuenta una ley suprema, pre-existente a la humanidad y al planeta que ella habita. (Bareto, 1962 : 84).

A partir de tales ideas, es fácil percibir las críticas de Tobías Barreto al pensamiento metafísico y a la teoría del derecho natural. Tales ideas atraviesan 
todo el trabajo del autor. Aliado al pensamiento de superación de estos ideales, Tobías Barreto también fue representante del cientificismo jurídico ${ }^{21}$ que permeó a esta nueva generación, así como también del evolucionismo (de la misma forma que Silvio Romero):

Lo que se quiere, y lo que importa principalmente, es hacer el derecho entrar en la corriente de la ciencia moderna, resumiendo, debajo de estas letras, los hallazgos más plausibles de la antropología darwiniana. Y esto no es solamente una exigencia lógica, es más una necesidad real para el cultivo del derecho; porqué no hay nada más pernicioso a las ciencias del hombre que mantenerlas enteramente aisladas (Bareto, 2001 : 11).

Francisco de Paula Batista fue otro importante autor y profesor de la Facultad de Recife, que va mas allá de las teorías del derecho natural; no obstante, no lo elimina del todo. En lo que difiere, en realidad, es en el modo como trata el derecho natural. Según Ricardo Marcelo Fonseca, lo que el autor entiende por 'derecho natural', "parece identificarse con una orden racional y universal, bien sea en los moldes de una razón subjetiva moderna que tanto se propalaba en este mismo periodo por Europa" (Fonseca, 2006b : 339-369). ${ }^{22}$ Aliado a esta nueva visión laica del derecho natural, se desprende también la necesidad del elemento científico en la actividad jurídica. En este sentido, encontramos en todos estos autores el cambio de referencial teórico desplazándose "en dirección a la búsqueda de un parámetro científico racional, que se va alejando, en fuerte tono polémico, de las anteriores teorías embebidas de una interpretación teológica de la ley (Fonseca, 2006b).

El próximo autor a ser estudiado es el jurista Augusto Teixeira de Freitas, ${ }^{23}$ que recibió influencia de Savigny y de la ideas alemanas. De hecho, Teixeira

21 Tal afirmación puede ser retirada del siguiente trecho de la obra del autor: "El espíritu científico tiene un principio regulador. Este principio es la Idea de desarrollo, concebido como ley que domina todos los fenómenos siderales y telúricos: seres de todas las especies inorgánicos y orgánicos, razas, pueblos, estados e individuos. Es en virtud de esta misma ley que el derecho, con todas sus apariencias de constancia e inmovilidad, también se encuentra como todo lo demás en un perpetuo tiempo, sujeto a un proceso de transformación perpetua. La inmutabilidad del derecho, ya sea como idea o como sentimiento, es una verdad temporal y relativa" (Fonseca, 2006b : 339-369). 22 Tal afirmación se encuentra en la obra del autor: "Algunos niegan la existencia del derecho natural, y dicen que el derecho positivo en sus lagunas se completa por sí mismo en virtud de su fuerza orgánica, derivada de los atributos de su universalidad y unidad, y de esta opinión es Savigny en su tratado de Derecho Romano $1^{\circ} § 46$. Pero, iqué es el derecho en su universalidad y unidad, si no el mismo derecho natural?" (Bareto, 2001 : 44).

23 Pontes de Miranda hace un resumen representativo de lo que significó la figura y la obra de Teixeira de Freitas para la cultura jurídica brasilera del siglo XIX: "Teixeira de Freitas fue, en el siglo XIX, el genio del derecho civil en América. La Consolidación de las Leyes Civiles, cuyas notas son magníficas fuentes de doctrina, y el Esbozo del Código Civil, representan lo que de mejor se 
de Freitas es conocido hasta los días de hoy como el mayor jurisconsulto del Imperio, por sus obras y por sus ideas, que influyeron las codificaciones y leyes esparcidas tanto en nuestro país como en las codificaciones extranjeras, entre ellas Argentina y algunas naciones latinoamericanas, elogiado por los autores de su época y los de hoy, como Lafayette, afirmando que los trabajos de este jurista "por la profundidad de las investigaciones, por la audacia del pensamiento y por la riqueza y erudición, compiten con lo mejor que se ha publicado en el extranjero" (Pereira, 1956 : 25). En los días, encontramos una importante obra que retrata no apenas la vida sino también las ideas de Teixeira de Freitas, del profesor Silvio Meira, bajo el título Teixeira de Freitas: el jurisconsulto del Imperio. (Meira, 1983). Es importante destacar también que a pesar de haber tenido una formación europea, Teixeira de Freitas consiguió adaptar y aplicara sus estudios observando, por encima de todo, las peculiaridades de la vida brasilera. ${ }^{24}$

De hecho, sus trabajos no se limitaron a una mera reproducción de lo extranjero; fue más allá, de tal forma que contribuyó para la formación de nuestra cultura jurídica. Tal hecho puede ser observado en la explicación presente en la introducción de la Consolidación: "examinar las leyes en sus propios textos sin influencia de opiniones ajenas, comparar atentamente las leyes nuevas con las antiguas, medir la precisión, el alcance y las consecuencias de unas y de otras, ahí se encuentra el laborioso proceso, que hemos empleado para conocer la substancia viva de la Legislación" (Freitas, 2003 : X) . ${ }^{25}$ Para la confección de la Consolidación, adoptó la sistemática alemana, dividiendo el trabajo en dos partes: una general y otra especial. Además, más allá de la sistemática de la legislación portuguesa, la obra de Teixeira de Freitas también fue marcada por la originalidad y la visión de futuro presente en su obra. El esbozo del Código Civil que, a pesar de no haber sido utilizado en nuestro país, fue aprovechado

tenía, en derecho civil, y que aparecieron después de 1860, y si bien es cierto es superior al Código Civil de 1916, fue ese proyecto malogrado para el Brasil y exitoso fuera de él, en Argentina, en Uruguay, en Paraguay. Muchas disposiciones que se encuentran en el Bürgesliches Gesetzbuch y en el Zivilgesetzbuch se encuentran en él; tal vez lo supieran los nuevos legisladores" (Miranda, $1981: 63)$.

24 Según Gilberto Freyre, tal hecho bastaría para que él mismo fuera considerado, al lado de José Bonifácio y Villa Lobos "máximos por su creatividad y por su brasileñidad, como autores de obras originales reconocidas por extranjeros. [...]. En la obra de Teixeira de Freitas, según Sílvio Meira, se revela 'el jurista, el observador, o hombre práctico'. Creo que podría haber calificado el jurista como jurista-sociólogo y como sociólogo del derecho -como futurólogo, hasta - a quien el tiempo social proyectado sobre el futuro procuró lidiar de modo tan filosófico-científico, como práctico. Pero sin que práctico significase para él apenas atender a lo inmediatamente útil [...]. Para Teixeira de Freitas -el casi sociólogo-, las leyes, siendo apenas legislativas, por encima de ellas estaban lo que llamó 'actos jurídicos' que, considerados en el contexto de sus ideas, serian actos jurídicos-sociólogos” (Meira, 1983 : xxxi).

25 La Introducción de la Consolidación es extremadamente rica y profunda, y deja entrever, por lo tanto, el carácter original de su trabajo. 
en otras naciones latinoamericanas, ${ }^{26}$ como mencionamos antes. Así, por toda esa significativa contribución, Teixeira de Freitas fue figura importante para la formación de nuestra cultura jurídica.

Otros profesores juristas y doctrinadores fueron importantes para el delineamiento de nuestra cultura jurídica en la segunda mitad del siglo XIX, tales como Lafayette Rodrigues Pereira, Perdigão Malheiros ${ }^{27}$ y Clóvis Beviláqua. Por hora, nos quedamos con estas cuatro figuras exponentes de su época, que fueron osados por quebrar las antiguas tradiciones dominantes en su tiempo. Sabemos que tal tarea de ruptura nunca es fácil, en la medida en que son precedidos por la fuerte resistencia de los tradicionalistas. Lo importante de estos autores es la contribución que los mismos ofrecieron para la formación de los contornos de nuestra cultura jurídica, yendo más lejos de las influencias extranjeras hasta entonces fuertemente arraigadas en nuestro ambiente jurídico.

Sin embargo, más allá de la importancia de la creación de las facultades de derecho instaladas en la primera mitad del siglo XIX y los juristas y profesores de la segunda mitad del siglo, otro factor importante que debe ser considerado al intentar comprender la formación de la cultura jurídica nacional consiste en el proceso de elaboración de la legislación en este periodo, que a continuación trataremos.

\section{La formación de la cultura jurídica y la producción legislativa independiente}

De hecho, después de la Independencia del Brasil, es importante recordar aquí que fue editada la Ley del 20 de octubre de 1823, determinando que continuasen en vigor las Ordenaciones, así como la legislación hasta entonces divulgada, hasta que no fuese confeccionado un Código o no fuesen alteradas. En seguida, la Constitución Imperial de 1824 determinó la necesidad de confeccionar un Código civil y Criminal, lo más pronto posible. Como se sabe, el Código Civil todavía demoraría mucho tiempo en ser organizado (solo en 1916); el Código criminal, al contrario, es promulgado en $1830,{ }^{28}$ y en $1850^{29}$

26 Silvio Meira apunta algunos países que fueron influenciados por el Esbozo de Código Civil en sus codificaciones: Argentina, Uruguay, Paraguay, Nicaragua, inclusive algunos países de Europa, como Alemania, Suiza, Italia, así como países del Oriente, como Japón y China (Meira, 1983).

27 Sobre Perdigão Malheiro, escribe Lafayette: "Los diversos escritos del Sr. Perdigão Malheiros se recomiendan por el estudio concienzudo y profundo de las materias, por la justicia de las ideas y por la concisión del estilo" (Pereira, 1956 : xxv).

28 Antonio Carlos Wolkmer refiere que el Código Criminal de 1830 "era no solo redactado según la mejor doctrina clásica penal, también, se afirmaba con el espíritu liberal de la época. Representaba un avance, si lo comparamos a los procesos crueles de las Ordenaciones" (Wolkmer, 2003 : 85-86). 29 Es interesante notar la organización del Código Comercial antes del Código Civil. Tal hecho es explicado por Antonio Carlos Wolkmer: "Las necesidades inmediatas, las actividades de negociación y el desarrollo comercial hicieron que la principiante burguesía latifundista priorizase la reglamentación de la vida económica sobre la vida civil. Teniendo en cuenta que, para la 
el Código Comercial. En este sentido, las Ordenaciones, que eran para ser algo provisional, continuaron entre nosotros hasta el inicio del siglo XX, con sus debidas cautelas dictadas por la "Ley de la Buena Razón", es claro.

Analizando la Constitución Imperial de 1824, un hecho que merece destaque es que, a pesar de la propuesta liberal, con influencia de la Revolución Francesa y del constitucionalismo francés, la misma no terminó con el régimen de trabajo esclavo (implantada en el Brasil por los portugueses, la esclavitud solo termina en 1888), reduciendo la persona

a la cosa, la renitencia de estructuras arcaicas en las propias instituciones político jurídicas, que se sumaron, a cada paso de las transformaciones institucionales brasileras, con una forma muy peculiar de adaptación de los principios liberales venidos sobre todo de Francia y de los Estados Unidos, siempre adecuados a los intereses de las elites (Fonseca, 2006b : 339-369).

Sin embargo, no obstante la permanencia de las Ordenaciones Filipinas entre nosotros, hubo, a partir de la Independencia, una creciente producción legislativa regulando más las relaciones privadas en nuestro país, por ejemplo, la "Ley de Tierras" (Fonseca 2005 : 97-112) de 1850, la reforma hipotecaria de 1864, (Varela 2005) el decreto 1144 de 1860 (que instituye el matrimonio no católico), y el decreto 181 de 1890, gran marco para el derecho de familia en el siglo XIX. Así, delante de tanta profusión legislativa, vamos dando contornos propios a nuestra cultura jurídica, distanciándose cada vez más de la antigua metrópoli.

En este sentido, era tal el volumen de la producción legislativa que en la segunda mitad del siglo XIX, cuando se inicia el proceso de codificación en nuestro territorio, sería necesario realizar un trabajo de Consolidación de la legislación vigente en nuestro país, trabajo este otorgado a Teixeira de Freitas, 42 seguido de la elaboración del Esbozo del Código Civil, iniciado en 1860 por Teixeira de Freitas, pero rescindido en 1872, sin que el Esbozo hubiese sido concluido, a pesar de haber servido de inspiración para diversas naciones, como fue mencionado.

\section{Consideraciones finales}

Así, a pesar de todo el esfuerzo de modernización jurídica realizado en este periodo, no conseguimos elaborar una codificación en el siglo XIX, lo que vendría a acontecer en el inicio del siglo XX. Sin embargo, tal hecho no es motivo para afirmar la permanencia inmóvil de la antigua legislación portuguesa

burguesía, ordenar el comercio y la producción de la riqueza era más imperiosa que la protección y la garantía de los derechos civiles, nada más natural que el Código Comercial precediese en 67 años al Código Civil (Wolkmer, 2003 : 88). 
en nuestro país, pues, como vimos, la profusión legislativa en el ámbito privado contribuyó tanto para regular los problemas específicos de nuestra sociedad como para, a partir de allí, delimitar los contornos de nuestra cultura jurídica en la segunda mitad del siglo XIX. En este sentido, podemos encontrar las efectivas contribuciones para la formación de la cultura jurídica brasilera a partir de varios profesionales del área jurídica, sean ellos juristas o profesores, así como las inúmeras leyes extravagantes editadas especialmente en la segunda mitad del siglo XIX.

\section{Referencias}

Adorno, S. (1988). Os aprendizes do poder. São Paulo: Paz e Terra.

Barreto, T. (2001). Estudos de Direito. Brasília: Senado Federal, Conselho Editorial.

Batista, F. de P. (1872). "Compêndio de hermenêutica jurídica para uso das Faculdades de direito do Império". Revista e consideravelmente aumentada. No. 3.

Benevides, J. M. C. de S. (1887). Philosophia elementar de direito público: interno, temporal e universal. São Paulo: s/ed.

Brotero, J. M. (1829). Princípios de Direito Natural compilados por José Maria de Avelar Brotero, lente do primeiro anno do Curso de S. Paulo. Rio de Janeiro: Tipografia imperial e nacional.

Carvalho, J. M. de (2002). Cidadania no Brasil: o longo caminho. Rio de Janeiro: Civilização Brasileira.

Cunha, L. A. (1980). A universidade temporã: o ensino superior da colônia à era de Vargas. Rio de Janeiro: Civilização brasileira.

Fonseca, R. M. (2006a). A cultura jurídica brasileira e a questão da codificação civil no século XIX, Revista da Faculdade de Direito. Universidade Federal do Paraná, Vol. 44, 61-76.

(2006b). "Os juristas e a cultura jurídica brasileira na segunda metade do século XIX". Quaderni fiorentini per la storia del pensiero giuridico moderno, Vol. 35, 339-369.

(2005). "A lei de terras e o advento da propriedade moderna no Brasil". Anuario Mexicano de História del Derecho. Tomo XVII. México: Instituto de Investigaciones Jurídicas. 
(2000). "A história no direito e a verdade no processo: o argumento de Michel Foucault”. Revista Gênesis de Direito Processual Civil, Vol 17. Hespanha, A. M. (2005). Cultura jurídica européia: síntese de um milênio. Florianópolis: Fundação Boiteux.

Meira, S. (1983). "Teixeira de Freitas: o jurisconsulto do Império". Revista e aumentada. No. 2.

Pereira, L. R. (1956). Direitos de família. Rio de Janeiro: F. Bastos.

Pontes de Miranda, F. C. (1981). Fontes e evolução do direito civil brasileiro. Rio de Janeiro: Forense.

Schwarcz, L. M. (1993). O espetáculo das raças: cientistas, instituições e questão racial no Brasil (1870-1930). São Paulo: Companhia das Letras.

Souza, J. S. de (1880). Elementos de philosophia do direito. Recife: Typografia central.

Texeira de Freitas, A. (2003). Consolidação das leis civis. Brasília: Senado Federal, Conselho Editorial.

Varela, L. B. (2005). Das sesmarias à propriedade moderna: um estudo de história do direito. Rio de Janeiro: Renovar.

Venâncio Filho, A. (1982). Das arcadas do bacharelismo. São Paulo: Perspectiva.

Wolkmer, A. C. (2003). História do direito no Brasil. Rio de Janeiro: Forense. 